\title{
EXPLORATION DU PLASMA SEMINAL HUMAIN : NOUVELLE APPROCHE PAR LA RESONANCE MAGNETIQUE NUCLEAIRE DU PROTON (RMN-'H)
}

\author{
S. Hamamah, F. Seguin*, C. Barthélémy, A. Fignon, S. Akoba*, A. Lepape*, J. Lansac, D. Royère \\ Unité de Biologie de la Reproduction, Dept. Gyn-Obs, CHU Bretonneau, 37044 Tours Cedex \\ * Laboratoire de Biophysique Cellulaire et RMN - INSERM U316, Faculté de Médecine, 37032 Tours Cedex
}

\section{HUMAN SEMINAL PLASMA : A NEW APPROACH TO ITS ANALYSIS USING PROTON NUCLEAR MAGNETIC} RESONANCE (PNMR). Human seminal plasma glycerylphosphorylcholine (GPC), glycerylphosphorylethanolamine (GPE), citric acid (Cit) and lactic acid (Lac) were analzed by measuring the peak area of proton nuclear magnetic resonance (NMR) spectra in four groups of patients : 21 men with secretory azoospermia ; 14 men with excretory azoospermia after vasectomy ; seven patients presenting with very severe oligoasthenozoospermia ; and 18 normozoospermic subjects. When comparing secretory and excretory azoospermia cases with normozoospermic men, seminal plasma GPC, Cit and Lac peak areas were lower in azoospermic cases compared to normozoospermic men $(16.79,8.18$ and 2.28 versus 23.38 , 10.58 and 4.3 arbitrary units respectively, $\mathrm{P}$ $<0.01)$. The peak area ratios $\mathrm{Cit} / \mathrm{Lac}$ and GPC/lac were significantly different between normozoospermic men and either secretory or excretory azoospermic groups $(\mathrm{P}<0.01)$. A significant difference was also found in the GPE/GPC peak intensity ratio between secretory and excretory azoospermia cases $(\mathrm{P}<0.001)$. These results demonstrate that quantitative markers such as GPC, GPE, Cit and Lac may be useful in the examination of human seminal plasma by proton NMR in infertility investigations. Keys words : NMR, seminal plasma, azoospermia, human. Andrologie 1992, 2 : 10-11.

\section{INTRODUCTION}

Le plasma séminal humain contient des sécrétions provenant à la fois des tubules séminifères, de l'épididyme et des glandes annexes.

A l'heure actuelle, l'exploration biochimique du plasma séminal repose surtout sur les dosages de certains marqueurs tels que le fructose pour les vésicules séminales, le zinc, les phosphatases acides, le citrate pour la prostate et la L-carnitine pour l'épididyme. Mais les informations fournies par ces dosages classiques sont parfois limitées et ne permettent pas dans certains cas d'établir un diagnostic précis, dû à une absence de standardisation (7).
L'utilisation de la résonance magnétique nucléaire dans l'exploration biochimique du plasma séminal des patients infertiles relance les voies de la recherche actuelle sur la biochimie du plasma séminal et permet d'avoir une approche plus quantitative, précise et reproductible.

Notre travail a pour but d'identifier et de quantifier par résonance magnétique nucléaire du proton (RMN-'H) des marqueurs biochimiques tels que la Glycérylphosphorylcholine (GPC), la Glycérylphosphoryléthanolamine (GPE), l'acide citrique (Cit) et l'acide lactique (Lac) dans le plasma séminal des azoospermies secrétoires, des oligo-asthénotératozoospermies extrêmes (OAT), des azoospermies excrétoires après vasectomie, et des témoins normospermiques.

\section{MATÉRIELS ET MÉTHODES}

4 groupes de patients ont fait l'objet de cette étude: 21 azoospermies sécrétoires, 14 postvasectomie, 7 OAT sévères et 18 normospermies. Les prélévements ont été effectués par masturbation après 3 jours d'abstinence. Après liquéfaction, un spermogramme a été réalisé (Tableau. 1). l'azoospermie a été confirmée par l'absence de spermatozoides dans le culot après une centrifugation $(750 \mathrm{~g}, 20 \mathrm{~min})$. Les plasma séminaux ont été congelés à - $20^{\circ} \mathrm{C}$ jusqu'à l'exploration en $\mathrm{RMN}-\mathrm{H}$.

Les spectres RMN du $1 \mathrm{H}$ ont été obtenus à $200 \mathrm{MHz}$ à partir de $0,5 \mathrm{ml}$ du plasma séminal dépourvu de débris cellulaires sur un spectromètre AM 200 WB (Bruker). Les acquisitions ont été réalisées après suppression du pic de l'eau par présaturation. Les mesures ont été réalisées par détermination de l'aire des pics sauf pour le rapport GPE/GPC où nous avons utilisé l'intensité des pics à cause de la proximité de ceux ci.

Les valeurs ont été représentées en termes de moyenne \pm SEM. Les comparaisons entre groupes ont été effectuées à l'aide des tests Anova et Mann et Whitney.

Tableau 1. Valeurs moyennes du Glycerylphosphorylcholine (GPC), de l'acide citrique (Cit) et de l'acide Lactique (Lac) dans le plasma séminal des azoospermies sécrétoires, excrétoires (Post-vasectomie) et des normospermies

\begin{tabular}{lllllll}
\hline \multirow{2}{*}{ Définition de la population } & \multirow{2}{*}{$\mathrm{N}^{\circ}$} & \multicolumn{2}{c}{ Numération } & & & \multicolumn{2}{c}{ Type de l'aire de pics } \\
\cline { 7 - 8 } & & spz/ml x $10^{6}$ & vol/ej. & GPC & Cit & Lac \\
\hline Azoospermie sécrétoire & 21 & 0 & $2.15 \pm 0.3$ & $16.79 \pm 1.16^{\mathrm{a}}$ & $8.18 \pm 0.79^{\mathrm{b}}$ & $2.28 \pm 0.11^{\mathrm{c}}$ \\
OAT sévère & 7 & $0.32 \pm 0.2$ & $5.22 \pm 0.4$ & $18.18 \pm 1.4$ & $10.2 \pm 1.16$ & $2.33 \pm 0.27^{\mathrm{c}}$ \\
Azoospermie excrétoire & 14 & 0 & $4.46 \pm 0.4$ & $19.72 \pm 1.54^{\mathrm{a}}$ & $9.58 \pm 1.31^{\mathrm{b}}$ & $2.43 \pm 0.18^{\mathrm{c}}$ \\
Normospermie & 18 & $74 \pm 13$ & $3.87 \pm 0.61$ & $23.38 \pm 1.38$ & $10.58 \pm 0.82$ & $4.3 \pm 0.34$ \\
\hline
\end{tabular}

$\mathrm{a}: \mathrm{P}<0.01$ comparé avec normospermic $-\mathrm{b}: \mathrm{P}<0.03$ comparé avec normospermic $-\mathrm{c}: \mathrm{P}<0.01$ comparé avec normospermic
Dans cette étude, nous avons comparé la GPC, le Citrate et le Lactate soit individuellement (Tableau 1), soit en termes du rapport GPC/Cit, $\mathrm{Cit} / \mathrm{Lac}, \mathrm{GPC} / \mathrm{Lac}$ et GPE/GPC dans tous les groupes (Fig. $1 \mathrm{~A}$ ). Une différence significative dans les rapports $\mathrm{Cit} / \mathrm{Lac}$, GPC/Lac et GPE/GPC a été trouvé entre le plasma séminal des azoospermies sécrétoires ou excrétoires par rapport au groupe témoin normospermique. Cependant, aucune différence n'a été observée pour le rapport GPC/Cit. Mais, seul le rapport d'intensité GPE/GPC montre une différence significative entre les azoospermie secrétoires et excrétoires (p<0,001; Fig. 1 B).

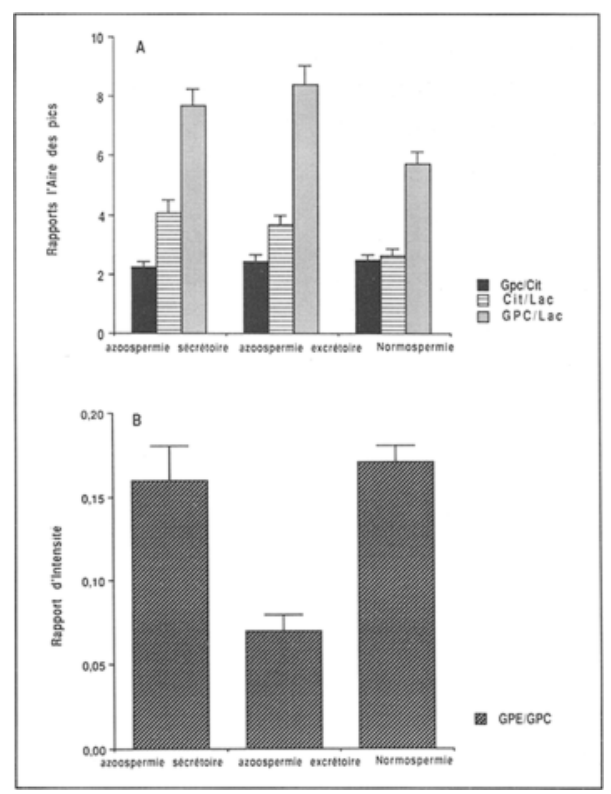

Fig. 1 A : Rapports de l'aire des pics GPC, Cit et Lact en RMN du $1 \mathrm{H}$ pour les azoospermies sécrétoires, excrétoires et les normospermies. Les valeurs sont représentées en moyenne $\pm S E M$.

Fig. 1 B. : Rapports de l'intensité des pics GPE/GPC en RMN $1 \mathrm{H}$ pour les azoospermies sécrétoires, excrétoires et les normospermies. Les valeurs sont représentées en moyenne \pm SEM. 


\section{DISCUSSION}

Si plusieurs études ont montré l'intérêt en RMN in vivo et permis l'évaluation de certaines fonctions testiculaire $(3,5)$, en revanche, peu ont été publiées sur l'usage de la RMN 31p pour étudier l'état fonctionnel de spermatozoides humain et animal $(1,4,6)$.

Dans notre étude, l'analyse des hormospermies en RMN-'H montre que l'aire de pics GPC, Cit et Lac du plasma séminal des normospermes est plus élevé que chez les azoospermes ou l'OATs. Il est important de noter qu'à l'exception de l'acide lactique, nous avons observé une grande variabilité inter-individuelle pour la GPC et le Cit. Nous avons confirmé que le plasma séminal des azoospermies sécrétoires, excrétoires et/ou des OAT contient moins d'acide lactique que celui de normospermie puisque le taux d'acide lactique est lié au nombre de spermatozoides de l'éjaculat.

Théoriquement, la GPC est synthétisée dans l'épididyme, par conséquent, on ne devrait pas en trouver dans le plasma séminal des patients après vasectomie. Mais, nous avons retrouvé un taux de GPC similaire à celui des azoospermies sécrétoires chez certains patients vasectomisés. Il est intéressant de noter que nous avons observé une différence significative pour le rapport d'intensité GPE/GPC entre les azoospermies sécrétoires et après vasectomies.

Ce rapport apparait donc comme un excellent paramètre pour déterminer le type de l'azoospermie au contraire de la transferrine séminale qui ne permet pas de différencier l'origine de l'azoospermie (2).
En conclusion, ce travail préliminaire démontre clairement l'importance de la $\mathrm{RMN}-\mathrm{-} H$ dans l'exploration du plasma séminal humain et fournit des données importantes dans le choix thérapeutique de l'hypofertilité masculine.

\section{REFERENCES}

1 - Bahl, S., Bawa, S.R., Srivastava S., Phadke, R.S, Govil G. : Magnetic resonance studies of intact human spermatozoa. Physiol. Chem. Phys and Med NMR.1988., 20: 183-188.

2 - Barthélémy C., Khalfoun B., Guillaumin J.M., Lecomte P. Bardos P. : Seminal fluid transferrin as an index of gonadal function in men. J. Reprod. Fert. 1988, 82: 113-118.

3- Bax, A. Two dimensional nuclear magnetic resonance in liquids. Ed. Delft University Press. 1984, Holland.

4 - Bretan, P.N., Vigneron, D.B., Hricak, H., Tom, R.A., Moseley, M., Tanagho, E.A James, T.L. : Assessment of male infertility: correlation between results of semen analysis and phosphorus-31 magnetic resonance spectroscopy. Urology. 1989, 33: 116-119.

5 - Chew, W.M Hricak, H. : Phosphorus-31 MRS of human testicular function and viability. Investigative Radiology. 1989, 24: 997-1000.

6 - Robitaille, P.M., Robitaille, P.A., Martin, P.A Brown, G. : Phosphorus-31 nuclear magnetic resonance studies of spermatozoa from the boar, ram, goat and bull. Comp. Biochem. Physiol. 1987, 87B: $285-296$.

7 - Soufir, J.C. : Azoospermie, asthénozoospermie et biochimie séminale. Ann. Biol. Clin. 1985, 43 : 67-70.
RÉSUMÉ. Nous avons identifié et évalué par Resonance Magnétique Nucléaire du Proton (RMN-'H), dans le plasma séminal humain, des marqueurs biochimiques tels que la Glycérylphosphorylcholine (GPC), la Glycérylphosphoryléthanolamine (GPE), l'acide Citrique (Cit) et l'acide Lactique (Lac). Les valeurs des marqueurs GPC, Cit et Lac sont plus faibles dans les groupes azoospermies sécrétoires, excrétoires ou OATs par rapport aux normospermies $(16,79 ; 8,18 ; 2,28$ vs 23,$38 ; 10,58$ et 4,3 respectivement; $p<0,01$ ). Les rapports de l'aire des pics $\mathrm{Cit} / \mathrm{Lac}$ et GPC/Lac montrent une différence significative entre les normospermies et les azoospermies sécrétoires ou excrétoires $(\mathrm{p}<0,01)$. Nous avons également trouvé une différence significative du rapport d'intensité GPE/GPC entre les azoospermies sécrétoires et excrétoires $(p<0,001)$. Cette étude nous a permis d'identifier des marqueurs tels que la GPC, la GPE, le Cit et le Lac. Ceux-ci peuvent être utilisés d'une part pour l'exploration du plasma séminal des patients infertiles et d'autre part pour differencier les azoospermies sécrétoires des excretoires. Mots clés: RMN, Plasma séminal, azoospermie, humain. Andrologie 1992, 2 : 10-11. 